Modelling the effect of pressure on the critical shear stress of $\mathrm{MgO}$ single crystals

\author{
J. Amodeo, Ph. Carrez and P. Cordier*
}

Unité Matériaux et Transformations,

CNRS-UMR 8207, Université Lille 1,

Cité Scientifique, Bâtiment C6, 59655 Villeneuve d'Ascq, France.

*corresponding author: Patrick.cordier@univ-lille1.fr

Published in Philisophical Magazine as :

Amodeo, J., Carrez, P., \& Cordier, P. (2012). Modelling the effect of pressure on the critical shear stress of MgO single crystals. Philosophical Magazine, 92(12), 1523-1541. 


\section{Modelling the effect of pressure on the critical shear stress of MgO single crystals}

\section{Abstract:}

We use a hierarchical multi-scale model to study the effect of high pressure on the critical shear stresses of MgO. The two main slip systems, $1 / 2<110>\{110\}$ and $1 / 2<110>\{100\}$ are considered. Based on a generalized Peierls-Nabarro model, we show that the core structure of $1 / 2<110>$ screw dislocations is strongly sensitive to pressure. Mostly planar and spread in $\{110\}$ at ambient pressure, the core of screw dislocations tends to spread in $\{100\}$ with increasing pressure. Subsequently, one observes an inversion of the easiest slip systems between 30 and $60 \mathrm{GPa}$. At high pressure, the plasticity of $\mathrm{MgO}$ single crystals is expected to be controlled by $1 / 2<110>\{100\}$ slip systems, except at high temperature where both slip systems become active. Pressure is also found to increase the critical resolved shear stresses and to shift the athermal temperature toward higher temperatures. Under high pressure, $\mathrm{MgO}$ is thus characterized by a significant lattice friction on both slip systems.

Keywords : Dislocation core modelling, kink-pairs mechanism, high pressure.

\section{Introduction}

The Earth's lower mantle (between 670 and $2900 \mathrm{~km}$ depths) is composed of silicate perovskites and of ferropericlase $\left(\mathrm{Mg}_{0.8} \mathrm{Fe}_{0.2}\right) \mathrm{O}$ as the second most abundant phase. Since the internal heat of the Earth is brought to the surface by solid-state convection in the mantle, the rheological behaviour of these phases is of primary importance for modelling the dynamics of our planet.

In a first approach, it is possible to leave the influence of the iron component aside and to concentrate on the intrinsic plastic properties of $\mathrm{MgO}$. Periclase $(\mathrm{MgO})$ is a ceramic stable under ambient pressure and its plasticity has been extensively studied [1-7]. In this oxide with the rock-salt structure, there is only one dislocation type involving $1 / 2<110>$ as a Burgers vector. These dislocations can glide in several planes: $\{110\},\{100\}$ or $\{111\}$ [8]. In MgO, the 
easiest glide system is $1 / 2<110>\{110\}$ followed at high temperature by $1 / 2<110>\{100\}[2,7]$. Recent studies [9] have shown that under nanoindenters, stress concentrations may locally trigger glide on $\{112\}$, without suggesting that large scale plasticity occurs on those planes. Evidence for glide on $\{111\}$ has been reported [10-13], but the occurrence of this slip system remains controversial.

In the Earth's mantle, minerals are subjected to extreme pressure and temperature conditions. Most important is the influence of pressure which ranges between 25 and $135 \mathrm{GPa}$ in the lower mantle. One of the major challenges is thus to understand the influence of pressure on the plasticity of lower mantle minerals. This question has been raised very early and in [14], Fontaine and Haasen suggested the possibility that electrostatic repulsion within stacking faults could have implications on the core structure of dissociated dislocations in alkali halides. Among alkali halides, it has been observed that the primary slip planes change from $\{110\}$ to $\{100\}$ with decreasing ionicity [8,15]. In [16], Karato suggested that pressure could affect electronic polarizability and hence changes the primary slip planes to $\{100\}$ at high pressure.

Performing deformation experiments under confining pressure has been a continuing effort in mineral physics. Below $1 \mathrm{GPa}$, confining pressure mostly prevents brittle failure $[4,17,18]$. The strength of $\mathrm{MgO}$ (at room temperature) under confining pressure to $25 \mathrm{GPa}$ has been inferred in a diamond anvil cell (DAC) from the lattice strains measured by X-ray diffraction [19]. These measurements have been extended to $40 \mathrm{GPa}$ by Meade and Jeanloz [20] and up to $227 \mathrm{GPa}$ by Duffy et al. [21]. More recently, Merkel et al. [22] and Tommaseo et al. [23] have used deformation experiments in the DAC to infer slip systems in $\mathrm{MgO}$ and ferropericlase from texture analysis. They conclude that $1 / 2<110>\{110\}$ remains the easiest slip system at high pressure. Recently, the development of the D-DIA apparatus has allowed deformation experiments to be performed up to 10-15 GPa with in situ measurements of stress and strain [24]. Although the pressure range is much smaller, compression experiments at room temperature in the D-DIA have suggested a smaller pressure influence on the strength of $\mathrm{MgO}$ compared to DAC experiments [25]. Further experiments [11,26] were performed under simultaneous high-pressure and high-temperature, leading to a first quantification of the pressure influence on the flow stress of $\mathrm{MgO}$ characterized by an apparent activation volume of $V^{*} \approx 2.410^{-6} \mathrm{~m}^{3} / \mathrm{mol}$ at $\mathrm{T}=1473 \mathrm{~K}$ and for a strain rate of $310^{-5} \mathrm{~s}^{-1}$ [26]. 
Alternatively, plasticity can be studied today by numerical modelling. Provided that the elementary mechanisms are properly described, this approach provides an attractive route to study the behaviour of materials under extreme conditions where experiments are difficult to perform. Several authors, including us, have addressed dislocation modelling in $\mathrm{MgO}$ [27-30]. In [29], we have performed a first study of dislocation properties based on the Peierls-Nabarro (PN) model. We have shown that using first-principles calculations of $\gamma$-lines (also called Generalized Stacking Faults energies) as input parameters of the PN model, the influence of pressure on the dislocation core structure can be taken into account. However, this study was limited to collinear dissociation and planar core structures, since only $\gamma$-lines could be introduced into the 1D-PN model. In particular, the screw dislocations exhibited distinct properties when introduced into different planes which was an obvious bias of the model. The second limitation of the PN model is that it does not consider the influence of thermal activation on plastic glide. In [31] we have shown that numerical multi-scale modelling can be used to reproduce plastic deformation of $\mathrm{MgO}$ at finite temperature with no adjusted parameters. In this previous study, we satisfactorily reproduced the evolution of experimental Critical Resolved Shear Stresses (CRSS) as a function of temperature. Since the electronic structure of $\mathrm{MgO}$ is taken into account through ab-initio calculations, this approach can be extended to describe the behaviour of $\mathrm{MgO}$ under pressure, as already shown in [29].

The goal of the present study is to investigate the influence of pressure and temperature on plastic deformation of $\mathrm{MgO}$, through the calculation of CRSS. In particular, we aim at clarifying the role of pressure on the primary glide plane. For that purpose, we use the Peierls-Nabarro Galerkin (PNG) method to model dislocation cores [32]. Since several potential glide planes (described by 2D $\gamma$-surfaces) are introduced simultaneously in the model, it is able to describe complex, potentially non-collinear or $3 \mathrm{D}$, dislocation cores in a realistic way. Then, we follow the hierarchical multi-scale model of Ref. [31] to evaluate the CRSS of MgO under pressures up to $100 \mathrm{GPa}$. Thermal activation of slip is modeled based on the kink-pair theory.

In the following, after a brief presentation of technical details of calculations, the results of dislocation core structures and Peierls stresses will be presented. Both screw and edge dislocation characters have been studied and are used to model the evolution of flow stress as a function of pressure and temperature. 


\section{Modelling Techniques}

As the modelling techniques, including dislocation core structure, dislocation mobility and CRSS calculations, have been presented in details in [31], we will only restate the key stages of the multi-scale approach.

\subsection{Dislocation core modelling}

Dislocation core modelling can be based either on fully atomistic simulations (based on either empirical potentials or electronic structure calculations) or on the Peierls-Nabarro (PN) model where the core structure emerges from the equilibrium in the core region between elastic and inelastic forces [33,34]. In this study, we use the PNG method [32,35] as implemented in the $\operatorname{Cod}^{2} e x$ software which offers the possibility to take multiple glide planes into account and to calculate complex (possibly three-dimensional) dislocation cores [36-39]. As in the initial PN model or in recent generalizations [40-43], the dislocation core structure is determined from the minimization of elastic energy (through an approximation of a continuous field representation) and an interplanar potential derived from $\gamma$-surfaces calculations. $\gamma$-surfaces represent the extra energy associated with any rigid-body shear in a given glide plane [44]. In the PNG approach [32,35], two distinct fields are used: (i) $u(r)$ is a three-dimensional displacement field which represents the continuous deformation around the dislocation core and (ii) $\phi(r)$ is a vector field which represents the displacement jump when crossing discontinuity surfaces characterized by $\gamma$-surfaces. Minimization with respect to $\phi$ is achieved by means of a time dependent GinzburgLandau equation whereas an element free Galerkin method is used to compute the evolution of $u(r)$. Once the equilibrium configuration of the dislocation core is reached, the finite element cell of PNG calculations can be strained in order to determine the Peierls stress, for which a dislocation displacement is irreversible in its glide plane.

In this study, the evolution of the electronic structure of $\mathrm{MgO}$ with pressure is captured through the calculation of the elastic constants tensor $C_{i j}$ and also through the $\gamma$-surfaces calculations performed with the VASP code [45], based on the density functional theory (DFT). All DFT calculations have been performed within the Generalised Gradient Approximation (GGA) and using the all-electrons Projector Augmented-Wave (PAW) method [46]. $\gamma$-surfaces calculations have been performed using the supercell technique $[47,48]$. Pressure is applied by fixing atomic 
layers on surfaces of the supercell [48] and by adjusting the cell volume according to the equation of state of $\mathrm{MgO}$ [49-51]. The initial atomic positions and cell parameters are taken for bulk energy minimization performed under 30,60 and $100 \mathrm{GPa}$ confining pressures. Extended details of the calculations, including calculations of unit cell parameters and elastic constants can be found in [29]. Table 1 summarizes the crystal cell parameters and the elastic constants tensor used throughout this study.

\subsection{Dislocation mobility and critical shear stresses}

The next step of the model is to calculate the mobility of dislocations at finite temperature. When temperature is increased above $0 \mathrm{~K}$, dislocation motion is thermally activated and, in a material with lattice friction such as $\mathrm{MgO}$ [52], the dislocation line moves from one Peierls valley (minimum energy position) to the next, through the nucleation and propagation of kink pairs $[8,27]$. In [31], we have shown that the mechanical properties of $\mathrm{MgO}$ can be reproduced by assuming that kink pair nucleation is the controlling stage. We thus follow the same approach here and use the Elastic Interaction (EI) method [53,54] to evaluate the critical energy $\Delta H^{*}$ for kink pair nucleation as a function of stress. In the EI method, $\Delta H$ results from three contributions: the elastic interaction energy $\Delta E$, the variation of the Peierls energy $\Delta P$ between a straight line and a kinked one and $W$, the plastic work of the applied stress $\tau$. Assuming a rectangular shape of height $h$ and width $w$ of the bow-out configuration at low stress, the change of enthalpy can be written as

$$
\Delta H=\Delta E+\Delta P-W
$$

where the three terms depend on the couple of variable $(h, w)$ as expressed in [8,53] or in [55] for the specific treatment of initially edge dislocations. Based on Equation (1), a critical shape $\left(h^{*}, w^{*}\right)$ of the bow out configuration and the associated critical enthalpy $\Delta H^{*}$ can thus be calculated in the saddle point configuration as a function of stress $\tau$ (usually limited to a quarter of the Peierls stress). In this study, we will strictly adopt the same formalism with a Peierls potential $V_{p}$ parameterised on dislocation core structure and Peierls stress calculated by PNG, as illustrated in section 3.5. 
Table 1. Lattice parameter $a$ and elastic constant tensor $C_{i j}$ as a function of pressure calculated using PAW pseudopotentials and GGA approximation. The anisotropy factor $A$ corresponds to $2 C_{44} /\left(C_{11}-C_{12}\right)$. The shear modulus $\mu$ and the Poisson ratios $v$ have been calculated for dislocation belonging to $1 / 2<110>\{110\}$ and $1 / 2<110>\{100\}$ slip systems respectively within the frame of the Stroh theory. Data presented for 0,60 or $100 \mathrm{GPa}$ can be found in [29] or [31].

\begin{tabular}{ccccccccc}
\hline \hline $\begin{array}{c}\text { Pressure } \\
(\mathrm{GPa})\end{array}$ & $a(\AA)$ & $\begin{array}{c}C_{11} \\
(\mathrm{GPa})\end{array}$ & $\begin{array}{c}C_{12} \\
(\mathrm{GPa})\end{array}$ & $\begin{array}{c}C_{44} \\
(\mathrm{GPa})\end{array}$ & $A$ & $\mu(\mathrm{GPa})$ & $\nu_{\{110\}}$ & $\nu_{\{100\}}$ \\
\hline 0 & 4.237 & 279 & 93 & 146 & 1.57 & 116.5 & 0.18 & 0.27 \\
30 & 4.038 & 538 & 138 & 170 & 0.85 & 184.4 & 0.23 & 0.20 \\
60 & 3.915 & 778 & 179 & 187 & 0.62 & 236.7 & 0.25 & 0.18 \\
100 & 3.799 & 1088 & 230 & 202 & 0.47 & 294.3 & 0.27 & 0.16 \\
\hline \hline
\end{tabular}

Following the nucleation of kink pairs, the average velocity [56] of a moving dislocation of length $L$ over a Peierls barrier of width $a^{\prime}$ can be written as

$v(\tau, T)=\frac{v_{D} a^{\prime} b L}{2 w^{2}} \exp \left(-\Delta H^{*}(\tau) / k T\right)$

Equation 2 corresponds to a modification to the original expression proposed by Dorn and Rajnak [57]. $v_{D} b / w$ is the vibration frequency of a thermal fluctuation of width $w$ on the dislocation of Burgers vector modulus $b . L / 2 w$ is the number of nucleation sites and the exponential term (where $k$ is the Boltzmann constant) corresponds to the probability of a successful forward jump under a given temperature $T$. This formalism is commonly used in Dislocation Dynamics codes [58] and has shown its efficiency to satisfactorily reproduce dislocation dynamics in the thermally activated regime [59-61], even at the atomic scale [62].

The thermally activated regime corresponds to a temperature range below a critical temperature (called the athermal temperature $T_{a}$ ), beyond which lattice friction is smeared out by thermal fluctuations. In [31], athermal temperatures $T_{a}$ were found to be $600 \mathrm{~K}$ and $1200 \mathrm{~K}$ for $1 / 2<110>\{110\}$ and $1 / 2<110>\{100\}$ respectively, in agreement with available experimental data at ambient pressure. Above $T_{a}$, plastic flow is dominated by interactions between dislocations. Below $T_{a}$, plastic flow is controlled by the intrinsic mobility of dislocations and the strain rate 
can be calculated from Orowan's equation (Equation (3)), assuming an average dislocation density of mobile dislocations $\rho$.

$\dot{\varepsilon}=\rho b v(\tau, T)$

where $b$ is the modulus of the Burgers vector and $v(\tau, T)$ is the dislocation velocity (equation 2) calculated for edge and screw characters. Throughout this study, we assume that the slowest character is the limiting factor. Thus the velocity of the slowest character is introduced in Orowan's equation. In the thermally activated regime, CRSS can be thus regarded as the stress required to satisfy equation (3) for a given strain rate value and at a given temperature.

\section{Results}

\section{$3.1 \gamma$-surfaces}
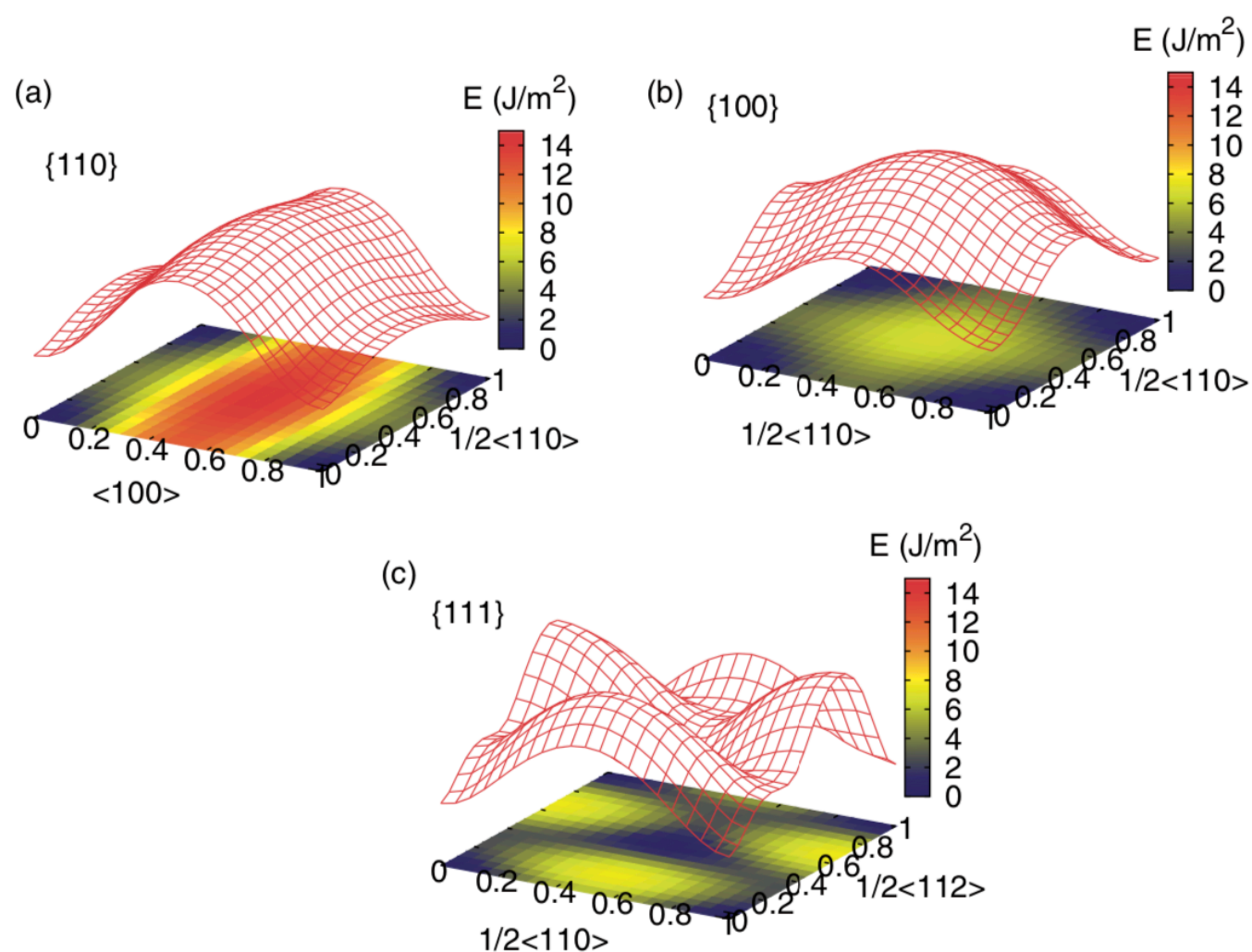

Figure 1. Typical $\gamma$ surfaces $\left(\mathrm{J} / \mathrm{m}^{2}\right)$ calculated at $60 \mathrm{GPa}$ : (a) $\{110\}$; (b) $\{100\}$; (c) $\{111\}$.

In order to model the core of $1 / 2<110>$ dislocations, we have calculated the $\gamma$-surfaces for the three $\{110\},\{100\}$ and $\{111\}$ planes which might be potentially sheared at the microscopic 
level. Each $\gamma$-surface has been calculated for the three pressures of interest i.e. 30, 60 and 100 GPa. As the overall shape of the $\gamma$-surfaces is unaffected by pressure, a typical example of these is given in Figure 1. Whatever the applied pressure, shear along $<110>$ corresponds to the easiest shear path for the three investigated planes, confirming that $1 / 2<110>$ is effectively the expected Burgers vector for dislocations in $\mathrm{MgO}$ whatever the pressure range. The strongest effect of pressure is nevertheless found on the $\gamma$-surfaces energy values (Figure 2). The values of unstable stacking faults at $1 / 4<110>$ are reported in Table 2. As already pointed out [29], these unstable stacking fault values increase when pressure increases. At low pressure, $\{110\}$ exhibits the lowest unstable stacking fault energy, whereas at 60 or $100 \mathrm{GPa}$, the $1 / 4<110>$ unstable stacking fault is lowest in $\{100\}$. Pressure also increases the $\{111\} \gamma$-surfaces (Figure $2 c$ ). At the same time, the $1 / 6<112>$ stacking fault (characteristic of closed packed planes in $f_{c c}$ lattices) tends to be stabilized as pressure reaches $100 \mathrm{GPa}$ with a staking fault energy of 3.05 $\mathrm{J} / \mathrm{m}^{2}$ (Figure 2d).

\subsection{Screw dislocations}
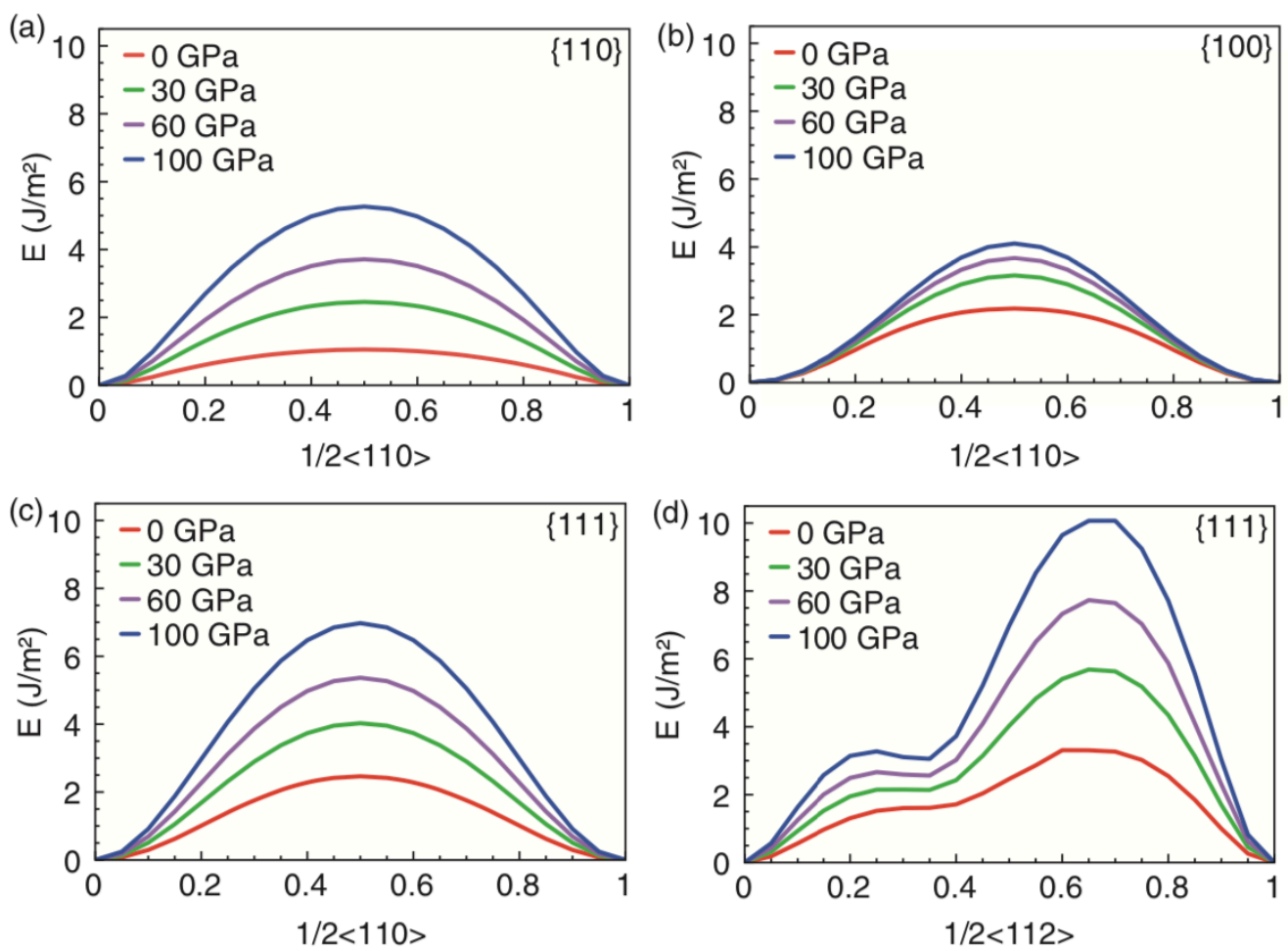

Figure 2. $\gamma$-line (along $1 / 2\langle 110\rangle$ ) evolution as function of pressure in $\{110\}$ (a), $\{100\}$ (b) and $\{111\}$ planes (c). (d) $1 / 2\langle 112\rangle \gamma$-lines in $\{111\}$ planes as function of pressure. 
Table 2. Unstable stacking fault energy $\left(\mathrm{J} \mathrm{m}^{-2}\right)$ corresponding to $1 / 4\langle 110\rangle$ in $\{110\},\{100\}$ and $\{111\}$ as a function of pressure. Data for $\{110\}$ and $\{100\}$ can be found in [29].

\begin{tabular}{lcccc}
\hline & $0 \mathrm{GPa}$ & $30 \mathrm{GPa}$ & $60 \mathrm{GPa}$ & $100 \mathrm{GPa}$ \\
\hline$\{110\}$ & 1.05 & 2.45 & 3.72 & 5.28 \\
$\{100\}$ & 2.18 & 3.16 & 3.67 & 4.11 \\
$\{11\}$ & 2.47 & 4.03 & 5.38 & 6.98 \\
\hline
\end{tabular}

In PNG calculations, screw and edge components are treated separately. We will first consider the case of screw dislocations for which the slip plane is not defined and which could, potentially, spread in any plane. The three $\{110\},\{100\}$ and $\{111\} \gamma$-surfaces as well as the elastic constants (Table 1) are used as input of $\operatorname{Cod}^{2} e x$ for the PNG calculations, with a PNG mesh homothetic to the crystal structure (see [31] for a complete description of the nodal mesh). After introduction of a Volterra dislocation in the simulation cell and relaxation of the core structure, the evolution of the vector field $\phi(r)$, monitored by the node displacements, gives access to the disregistry within the dislocation core. Whatever the pressure, screw dislocations remain pure screw in character. However, spreading of the core within a particular plane evolves. In Figure 3, we plot the node displacement associated with $\phi(r)$ along the Burgers vector direction for two crossing $\{110\}$ and $\{100\}$ planes containing the dislocation line. To quantify the amount of fractional dislocation spread into $\{110\}$ and $\{100\}$, we have fit the displacement using an arctangent function $\phi_{l}$ (Equation (4)) as is usually done in the PN model.

$\phi_{i}=\frac{b_{i}}{\pi} \arctan \frac{x}{\varsigma_{i}} \pm \frac{b_{i}}{2}$ 

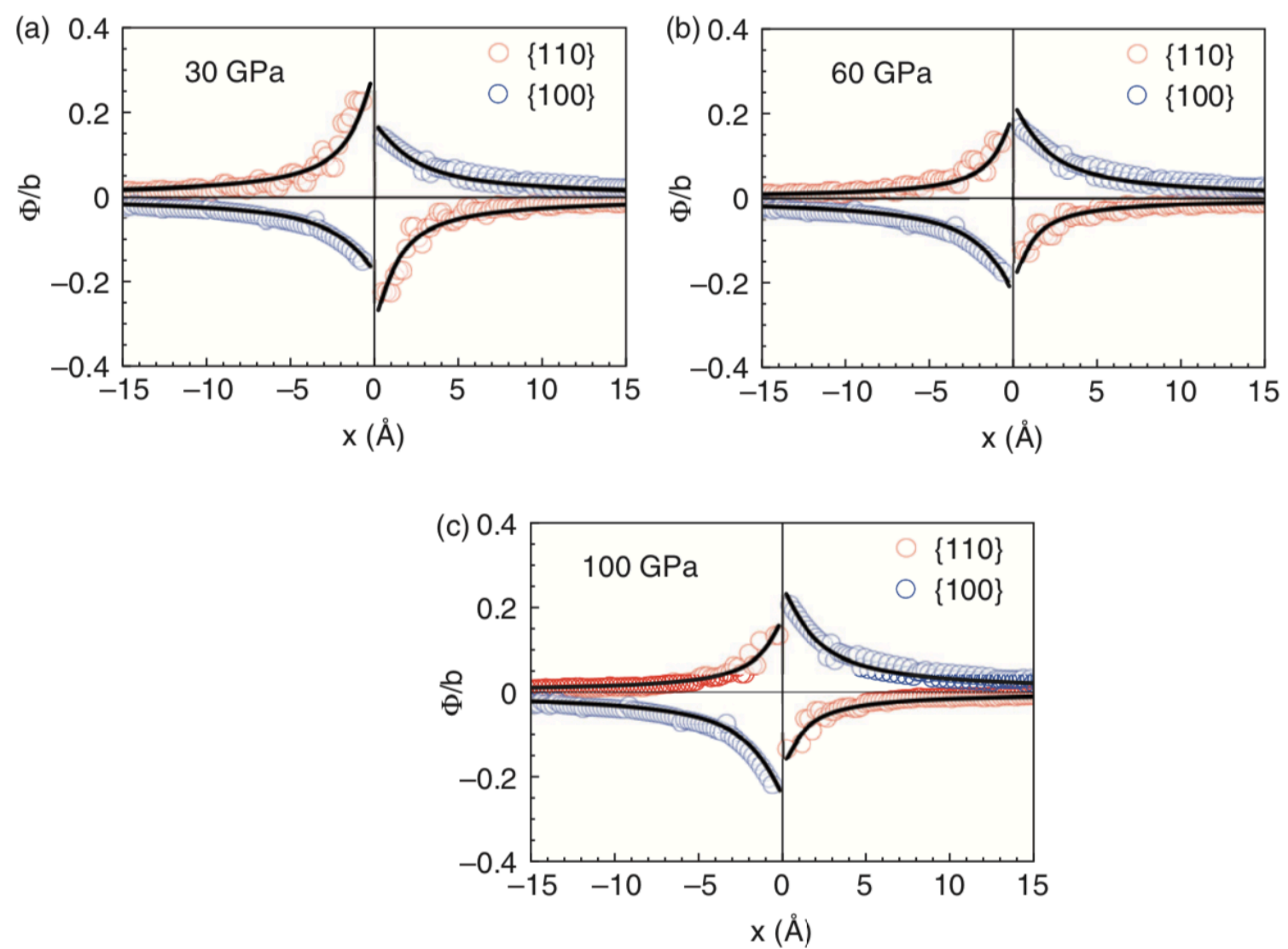

Figure 3. Evolution of the fractional distribution of Burgers vector of screw $1 / 2\langle 110\rangle$ dislocation $\{110\}$ and $\{100\}$ as a function of pressure: (a) $30 \mathrm{GPa}$; (b) $60 \mathrm{GPa}$; (c) $100 \mathrm{GPa}$. Trend lines correspond to a fit using Equation (4).

For a perfect screw dislocation of Burgers vector $b$, restricted to a single glide plane, $\phi$ would evolve from 0 to $b / 2$ and from $-b / 2$ to 0 as the glide plane is crossed. Here, $\phi_{l}$ corresponds to the disregistry in the $i$ plane ( $i$ stands for $\{110\},\{100\}$ or $\{111\}$ planes, $x$ corresponds to a position coordinate from the dislocation centre in the $i$ plane), $b_{i}$ is the amount of Burgers vector spread into the $i$ plane and $\zeta_{i}$ is the half-width of the Burgers vector density. The results of Equation (4) are plotted in Figure 3 and the key features, $b_{i}$ and $\zeta_{i}$ are given in Table 3.

Screw dislocations, calculated at 30,60 and $100 \mathrm{GPa}$, are found to spread mostly and simultaneously in $\{110\}$ and in $\{100\}$. Whereas the fraction of $b$ in $\{110\}$ reaches $60 \%$ at low pressure, it drops to $35 \%$ at the highest pressure. At the same time, the fraction of $b$ in $\{100\}$ evolves from $35 \%$ to $50 \%$. The remaining $15 \%$ of the Burgers vector is distributed in the two $\{111\}$ planes $(7 \%$ in each) containing the $1 / 2<110>$ direction.

\subsection{Edge dislocations}


Table 3. Key parameters for screw dislocation cores as a function of pressure. Fractional dislocation core $b_{i}$ are given in $\% b$ distributed in $\{110\}$ and $\{100\}$ planes. $\zeta$ correspond to the core size of fractional and Peierls stress for dislocation gliding in $\{110\}$ and $\{100\}$. Data given at $0 \mathrm{GPa}$ of pressure are taken from [31]. At low pressure (i.e. 0 or $30 \mathrm{GPa}$ ) fractional amount of Burgers vector spread in $\{111\}$ is negligible, at higher pressure (i.e. 60 and $100 \mathrm{GPa}$ ) only a few $15 \%$ of $b$ is expected in $\{111\}$ planes.

\begin{tabular}{lccccccc}
\hline & \multicolumn{3}{c}{110} & & \multicolumn{3}{c}{100} \\
\cline { 2 - 3 } \cline { 7 - 8 } Pressure $(\mathrm{GPa})$ & $b_{i}(\% b)$ & $\zeta(\AA)$ & $\tau_{P}(\mathrm{MPa})$ & & $b_{i}(\% b)$ & $\zeta(\AA)$ & $\tau_{P}(\mathrm{MPa})$ \\
\hline 0 & 80 & 1.7 & $150( \pm 70)$ & & 20 & 3.2 & $1600( \pm 220)$ \\
30 & 60 & 1.4 & $600( \pm 140)$ & & 35 & 2.3 & $750( \pm 120)$ \\
60 & 40 & 1.2 & $1300( \pm 210)$ & & 45 & 2 & $750( \pm 150)$ \\
100 & 35 & 1.4 & $1950( \pm 300)$ & & 50 & 2 & $850( \pm 150)$ \\
\hline
\end{tabular}
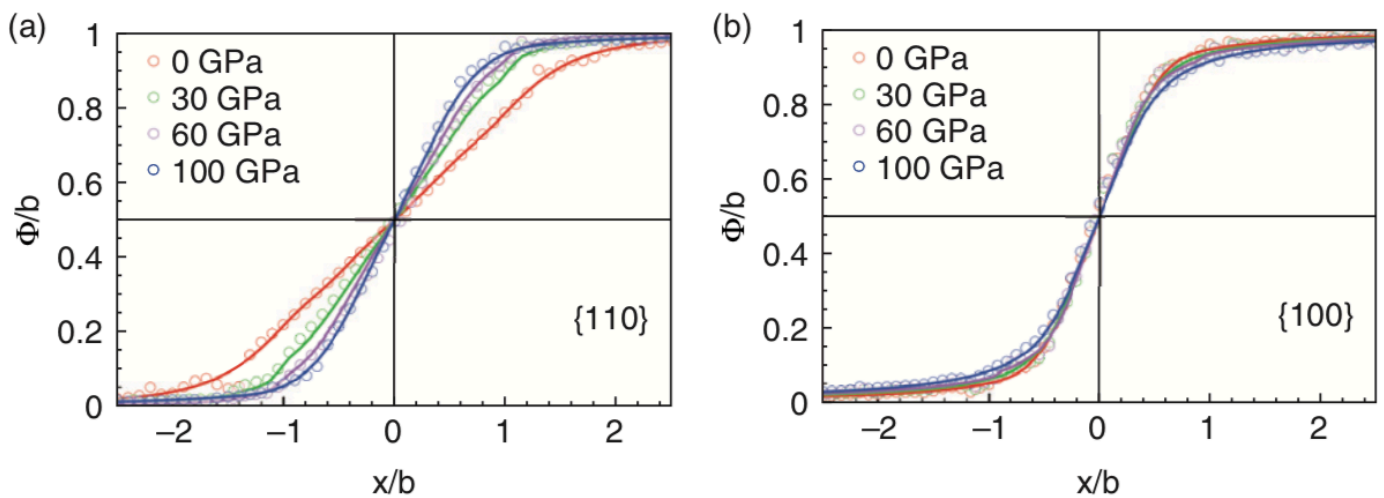

Figure 4. Evolution of the disregistry function for edge dislocation core as function of pressure for $1 / 2\langle 110\rangle\{110\}$ (a) and $1 / 2\langle 110\rangle\{100\}$ (b).

Since screw dislocations spread mostly in $\{100\}$ and $\{110\}$ and very little in $\{111\}$, their glide in $\{111\}$ will be extremely difficult. For this reason, $1 / 2<110>$ edge dislocations will be calculated in the $\{110\}$ and $\{100\}$ planes only. Calculations are performed at $0,30,60$ and 100 $\mathrm{GPa}$.

For $1 / 2<110>\{110\}$ and $1 / 2<110>\{100\}$ slip systems, edge dislocations exhibit planar cores. The shear profiles across the cores are presented in Figure 4. For both slip systems, we note that pressure tends to reduce core spreading (Table 3). However, when core spreading is scaled to $b[62]$, the reduced size $\zeta / b$ of $1 / 2<110>\{100\}$ edge dislocations remains roughly constant (around 0.5 , representing a narrow dislocation core $[63,64]$ ). The core of $1 / 2<110>\{110\}$ edge dislocations show the strongest sensitivity to pressure with a transition from a wide core $(\zeta / b>$ 1) at ambient pressure to a narrow core $(\zeta / b<1)$ as soon as pressure reaches $60 \mathrm{GPa}$. 


\subsection{Peierls stress calculations}

Table 4. Key parameters for edge dislocation cores as a function of pressure. Core widths are given in (A) and also in fraction of $b$ (in parentheses). Core width determined using a 1D PN model [29] are also given for comparison purpose between the two method.

\begin{tabular}{lccccccc}
\hline & \multicolumn{3}{c}{$1 / 2\langle 110\rangle\{110\}$} & & \multicolumn{3}{c}{$1 / 2\langle 110\rangle\{100\}$} \\
\cline { 2 - 3 } \cline { 8 - 9 } Pressure $(\mathrm{GPa})$ & $\zeta(\AA)$ & \multicolumn{1}{c}{$\zeta_{\mathrm{PN}}(\AA)$} & $\tau_{P}(\mathrm{MPa})$ & & $\zeta(\AA)$ & $\zeta_{\mathrm{PN}}(\AA)$ & $\tau_{P}(\mathrm{MPa})$ \\
\hline 0 & $3.6(1.2)$ & 5.2 & $80( \pm 30)$ & & $1.6(0.54)$ & 2.2 & $300( \pm 100)$ \\
30 & $2.8(1.0)$ & 3.3 & $310( \pm 190)$ & & $1.5(0.55)$ & 1.6 & $570( \pm 200)$ \\
60 & $2.4(0.8)$ & 2.6 & $760( \pm 300)$ & & $1.3(0.46)$ & 1.5 & $640( \pm 360)$ \\
100 & $1.9(0.7)$ & 2.2 & $620( \pm 170)$ & & $1.3(0.50)$ & 1.4 & $920( \pm 220)$ \\
\hline
\end{tabular}

When the relaxed structure of the dislocation core is determined, the PNG simulation cell is strained in order to evaluate the Peierls stress $\tau_{P}$ i.e. the threshold stress (or strain) for the dislocation to move from the initial relaxed configuration. Peierls stress evaluations have been performed for $1 / 2<110>\{110\}$ and $1 / 2<110>\{100\}$ dislocations (for screw and edge components). The calculated Peierls stresses can be found in Tables 3 and 4. As defined as a threshold stress, it is important to note that these values can be regarded as upper limit, in particular for edge dislocations. Nevertheless, two distinct conclusions can be drawn. For $1 / 2<110>\{110\}$, Peierls stresses increase monotonically with increasing pressure for both characters. Peierls stresses for edge dislocations are always found below those of screws. For $1 / 2<110>\{100\}$, we observe a different behaviour with an increase of Peierls stresses for edge dislocations with increasing pressure whereas, Peierls stresses for screw dislocations remain roughly constant with pressure.

\subsection{Dislocation mobility at finite temperature and CRSS}

Kink pairs nucleation is the mechanism which allows a dislocation to overcome Peierls barriers in the thermally activated regime. Nucleation energy of kink pairs (Equation (1)) is then one of the key parameters governing the dislocation velocity at a given temperature. CRSS can be thus evaluated below $T_{a}$ by coupling Equations (2) and (3) as described in section 2.

In Equation (1), the expression of the plastic work $W=b h w \tau$ is trivial, but the expressions of $\Delta E$ and $\Delta P$ require more considerations. In particular, the self-energy of the dislocation is 
controlled by a cut-off length [53]. For sake of consistency between different studies, we parameterized this cut-off length on the half-width of the dislocation core [31] calculated by the classical PN approach (i.e. when the whole Burgers vector density is spread within the glide plane [29]). The shear modulus and Poisson ratio appearing in the expression of $\Delta E$ are given in Table 1. One may note that two Poisson ratios are considered to take anisotropic effects into account at this stage of the calculation. The expression of $\Delta P$ is obviously strongly dependent on the Peierls potential $V_{P}$ expressed as a function of the position $x$ along the Peierls potential. Here, without any robust information about the shape of the potential, we parameterized all Peierls potential functions using Equation (5) (a modified version of $V_{P}$ proposed in [53]) where the Peierls stresses $\tau_{P}$ (given in Tables 3 and 4) and also the fractional Burgers vector $b_{i}$ spread in a given plane are taken into account, through $\alpha=\left(1-b_{i}\right) / 2$.

$$
V_{P}(x)=\frac{C(\alpha)}{2 \pi} a^{\prime} b \tau_{P}\left(1-\cos \left(\frac{2 \pi x}{a^{\prime}}\right)-\frac{\alpha}{2}\left(1-\cos \frac{2 \pi x}{a^{\prime}}\right)^{2}\right)
$$

and

$$
C(\alpha)=\sqrt{2} \frac{(1-\alpha)^{2}+4 \alpha^{2}+(1-\alpha) \sqrt{(1-\alpha)^{2}+8 \alpha^{2}}}{\left((1-\alpha)^{2}+2 \alpha^{2}+(1-\alpha) \sqrt{(1-\alpha)^{2}+8 \alpha^{2}}\right)^{3 / 2}}
$$

Equation (5) allows us to vary the shape of the Peierls potential. Practically, for a pure planar dislocation, such as edge dislocations, $b_{i}=1, \alpha=0$, and Equation (5) corresponds to a simple sinusoidal shape. For screw dislocations, the Peierls potential evolves from a steep shape in case of small fractional amount of dislocation ( $\alpha$ may reach the maximum value of 0.5 for a null spreading of $b$ in a given plane) to a close sinusoidal shape for motion in the dominant glide plane (i.e the largest amount of fractional dislocation is found in the plane and $b_{i}$ tends to $1)$.

The evolution of $\Delta H^{*}$ as a function of applied stress is presented in Figure 5. Values of $\Delta H^{*}(\tau=0)=\Delta H_{0}$, corresponding to twice the energy of an isolated kink, are presented in Table 5 , as well as the critical value of the kinks pair width $\mathrm{w}_{\mathrm{c}}$. Indeed, the kinks pair width $w^{*}$ shows a strong decrease at low stress before reaching a constant value $\mathrm{w}_{\mathrm{c}}$.

The EI method is restricted to low stresses and we performed the calculations for applied stresses up to $0.2 \tau_{P}$. As a consequence, data points of Figure 5 have been used to extrapolate 
the $\Delta H^{*}(\tau)$ curves using a classical Kocks formalism [65]. The results of this extrapolation are given as a trend line in Figure 5. Two distinct features are observed. For $1 / 2<110>\{110\}$, kink pair nucleation enthalpy for screw dislocations is higher than for edge, whatever the pressure. This can be interpreted as evidence that screw dislocations control plastic flow. For $1 / 2<110>\{100\}$, kink pair nucleation enthalpies are closer and, at high pressure, edge dislocations become the controlling components.

At this point, the key parameters governing the dislocation velocity (Equation (2)) are determined. In the thermally activated regime, interactions between defects are weaker than lattice friction [66]. The mobile dislocation density considered in the Orowan equation can be thus considered as the total dislocation density $\rho$. The typical length $L$ of moving dislocation is scaled to $1 / \sqrt{ } \rho$. Combining Orowan equation (Equation (3)) and Equation (2) leads to the following expression of strain rate $\dot{\varepsilon}$ (Equation (7)).

$\dot{\varepsilon}=\sqrt{\rho} \frac{v_{D} a^{\prime} b^{2}}{2 w_{c}^{2}} \exp \left(-\Delta H^{*}(\tau) / k T\right)$

The CRSS as a function of pressure is thus deduced from Equation (7), considering a typical constant strain rate of $10^{-4} \mathrm{~s}^{-1}$ and a typical dislocation density $\rho$ of $10^{12} \mathrm{~m}^{-2}$. The results are given in Figure 6 (ambient pressure calculations from [31] are reproduced for comparison with the present calculations performed at 30,60 and $100 \mathrm{GPa}$ ). Under such conditions, up to 30 $\mathrm{GPa}$, the deformation is governed by $1 / 2<110>\{110\}$ which exhibits the lowest CRSS. A change in dominant slip system in favour of $1 / 2<110>\{100\}$ is then observed for pressures higher than $30 \mathrm{GPa}$ as presented on Figure $6 \mathrm{c}$ and $6 \mathrm{~d}$. 
$1 / 2<110>\{110\}$
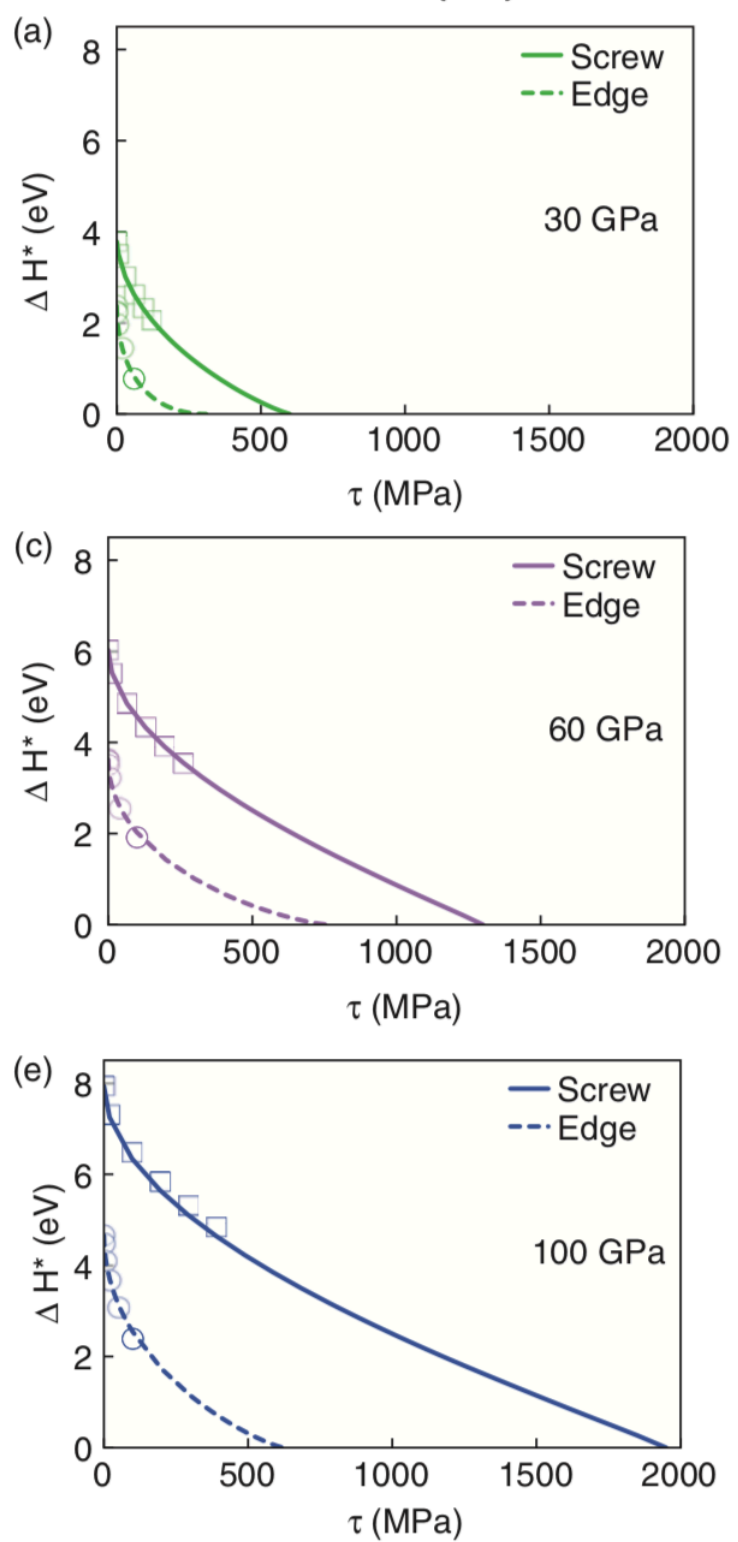

$1 / 2<110>\{100\}$
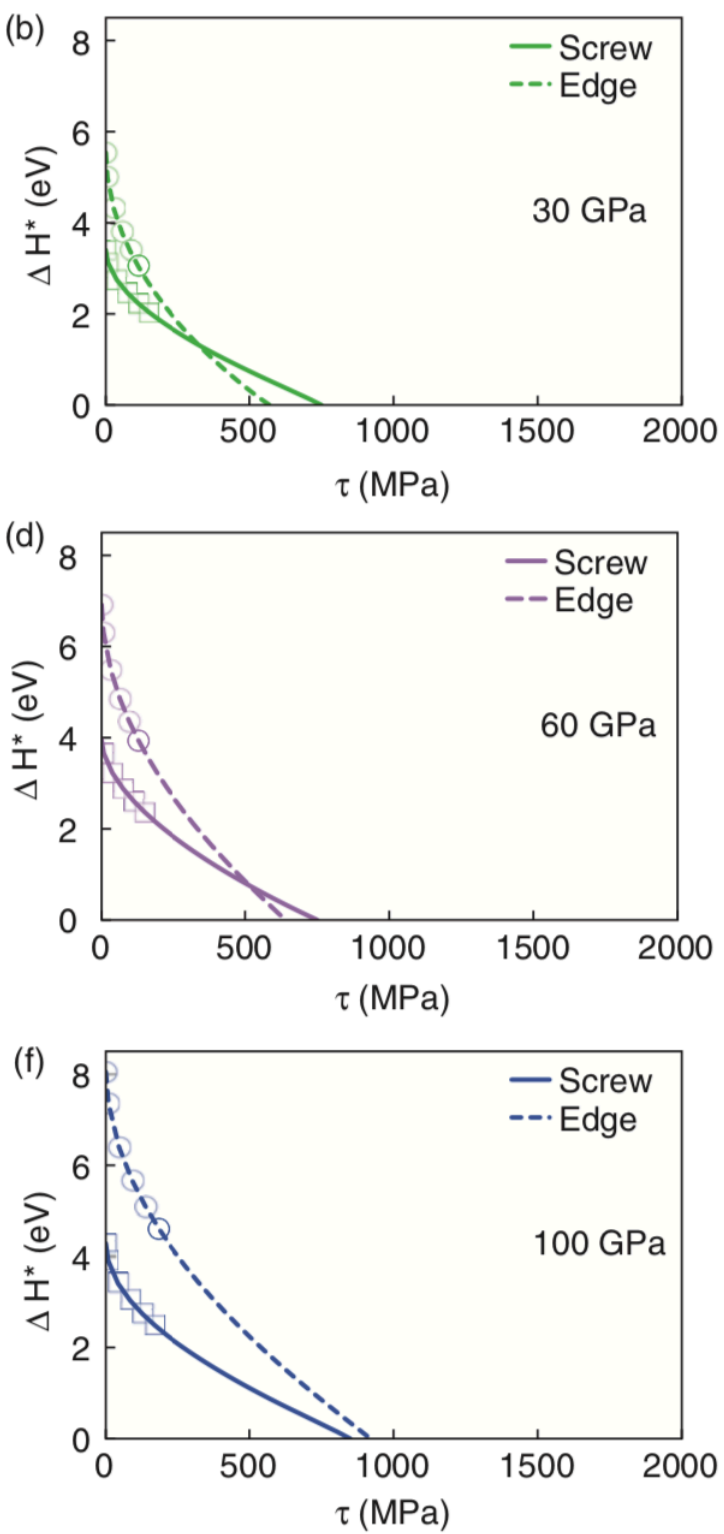

Figure 5. Critical kink pair nucleation enthalpy $\Delta H^{*}(\tau)$ as a function of pressure for $1 / 2\langle 110\rangle\{110\}(30 \mathrm{GPa}$ (a), $60 \mathrm{GPa}$ (c), $100 \mathrm{GPa}(\mathrm{e}))$ and for $1 / 2\langle 110\rangle\{100\}$ (30 GPa (b), $60 \mathrm{GPa}$ (d), $100 \mathrm{GPa}(\mathrm{f}))$.

Table 5. Values of $\Delta H_{0}(\mathrm{eV})$ and $w_{c}$ (in units of $b$ ) as a function of pressure for $1 / 2\langle 110\rangle\{110\}$ and $1 / 2\langle 110\rangle\{100\}$ screw and edge dislocations.

\begin{tabular}{|c|c|c|c|c|c|c|c|c|c|}
\hline & & \multicolumn{2}{|c|}{$P=0 \mathrm{GPa}$} & \multicolumn{2}{|c|}{$P=30 \mathrm{GPa}$} & \multicolumn{2}{|c|}{$P=60 \mathrm{GPa}$} & \multicolumn{2}{|c|}{$P=100 \mathrm{GPa}$} \\
\hline & & $\Delta H_{0}$ & $w_{c}$ & $\Delta H_{0}$ & $w_{c}$ & $\Delta H_{0}$ & $w_{c}$ & $\Delta H_{0}$ & $w_{c}$ \\
\hline \multirow[t]{2}{*}{$1 / 2\langle 110\rangle\{110\}$} & screw & 1.18 & 110 & 3.89 & 60 & 6.04 & 42 & 7.95 & 40 \\
\hline & edge & 0.68 & 100 & 2.38 & 90 & 3.65 & 50 & 4.70 & 80 \\
\hline \multirow[t]{2}{*}{$1 / 2\langle 110\rangle\{100\}$} & screw & 2.57 & 20 & 3.40 & 55 & 3.95 & 80 & 4.29 & 80 \\
\hline & edge & 2.92 & 70 & 5.55 & 70 & 6.98 & 80 & 8.12 & 60 \\
\hline
\end{tabular}



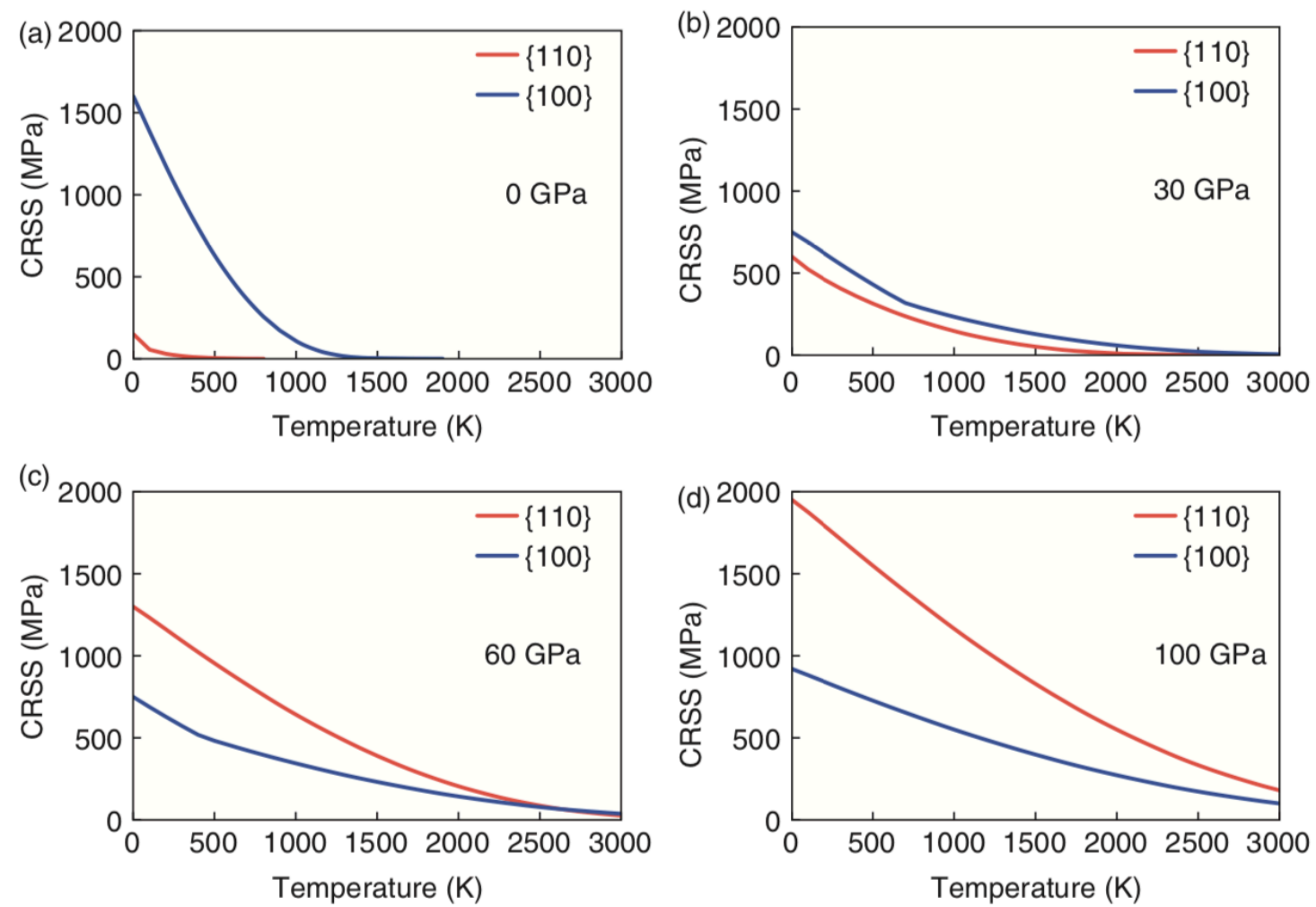

Figure 6. Critical resolved shear stress versus temperature for both $1 / 2\langle 110\rangle\{110\}$ (red curve) and $1 / 2\langle 110\rangle\{100\}$ (blue curve) as a function of pressure: (a) ambient pressure; (b) $30 \mathrm{GPa}$; (c) $60 \mathrm{GPa}$; (d) $100 \mathrm{GPa}$.

\section{Discussion}

Confining pressure has been commonly used in rock mechanics to prevent brittle failure. The pressure range involved was relatively small (typically a few hundreds of MPa) and thus did not affect significantly the intrinsic properties of rocks and materials. This is not the case for the properties of materials in the context of planetary interiors. Let us consider here $\mathrm{MgO}$ within the Earth's mantle as a case study. Compression of this oxide to $100 \mathrm{GPa}$ corresponds to an excess free energy of $0.6 \mathrm{eV} / \mathrm{atom}$. The electronic structure and bonding of $\mathrm{MgO}$ are clearly modified in the pressure range experienced in the Earth's mantle. This can be illustrated also from the elastic properties. Table 1 shows that the pressure range of the mantle is of the same order of magnitude as the elastic constants. Consequently, a compression under $100 \mathrm{GPa}$ results in a four fold increase of $C_{11}$ (a factor of 2 increase for $C_{12}$ and 1.4 for $C_{44}$ ). This is the first and most trivial effect of pressure which reflects the anharmonicity of the crystal. Most plastic properties (like the nucleation of dislocations through Frank-Read sources, long-range elastic interactions or lattice friction) scale to the elastic properties (usually through a shear modulus) 
and it is expected that plastic properties will evolve with pressure as the elastic constants or shear moduli do.

Since plastic deformation involves crystal defects, one must also consider the influence of pressure on the structure and mobility of these defects. This question has been addressed very early by Fontaine and Haasen [14] who attributed the pressure effect observed on $\mathrm{NaCl}$ to the pressure-induced change of the dissociation width of dislocations in the $\{110\}$ planes. This approach has been extended by Belzner and Granzer [67] who calculated at the atomic level the influence of pressure on the dissociation width of an edge $1 / 2<110>\{110\}$ dislocation. More recently, some studies have addressed this effect of pressure on dislocation cores and mobility [68-70]. However, this field remains largely unexplored.

Our approach based on the PN model follows the route opened by Fontaine and Haasen [14]. Since the PN model describes the interplay between elastic and inelastic forces in shaping the dislocation core, it is well suited to highlight their respective roles. Indeed, the $\gamma$-surfaces used as an input for the PNG model already bring a lot of information. Their overall trend is, as elastic constants, to increase with increasing pressure. However this effect is differential and the evolution with pressure of the $\{110\} \gamma$-surfaces is much faster than the one of $\{100\} \gamma$ surfaces. An inversion of easy glide systems with pressure can be anticipated qualitatively at this stage. It is necessary to go further and to consider the dislocation cores models.

Let us first discuss the case of the planar cores of edge dislocations. The core spreading of $1 / 2<110>\{100\}$ edge dislocations decreases with increasing pressure. To assess the respective role of elastic and non-elastic components, one has to consider the reduced core width $\zeta / b$. In doing so, the core spreading appears to be insensitive to pressure (Figure $4 \mathrm{~b}$ ) suggesting that this dislocation responds elastically to the applied pressure. Consequently, Peierls stresses scale around a constant value of $210^{-3} \mu$. However the core width of $1 / 2<110>\{110\}$ edge dislocations evolves, especially between 0 and $30 \mathrm{GPa}$. This evolution is visible on the normalized core profile (Figure 4a), demonstrating that it is not merely an elastic effect. At ambient pressure, the core of $1 / 2<110>\{110\}$ edge dislocations is wide, resulting in a low lattice friction (Peierls stresses: $510^{-4} \mu$ ). At high pressure (above ca. $60 \mathrm{GPa}$ ), their cores become narrow and consequently lattice friction increases significantly (Peierls stress of the order of 2 $10^{-3} \mu$ between 60 and $100 \mathrm{GPa}$ ). 
A more pronounced effect of pressure is found on screw dislocation cores. These changes are related to the fact that screw dislocations are not ascribed to a given glide plane. The differential behaviour of the $\gamma$-surfaces as a function of pressure results in a differential tendency of the core to spread shear in a 3D structure which is well-captured by the PNG model (contrary to the PN model, this aspect is further discussed below). Whereas at ambient pressure, $80 \%$ of the Burgers vector is spread in $\{110\}$, we find that, at $100 \mathrm{GPa}$, the core of the screw dislocation is mostly spread in $\{100\}$. Following this structural evolution, the associated Peierls stresses increase continuously in $\{110\}$ and decrease in $\{100\}$ as pressure increases.

Our results show also that under pressure, the only favoured glide planes are $\{100\}$. The tendency for stabilizing the dissociation of $1 / 2<110>$ edge dislocation in $\{111\}$ into $1 / 6<112>$ partials dislocations is suggested by the evolution of the $\gamma$-surfaces (Figure 2d). Indeed, we have previously shown that PNG calculations predict this Shockley partials dissociation in $\{111\}$ (calculations at $100 \mathrm{GPa}$ presented in [55]). However, since the screw dislocation does not tend to spread significantly into $\{111\}$, the corresponding slip system will remain hard. The indirect evidences of $\{111\}$ as potential slip planes, in addition to $1 / 2<110>\{100\}$, in some high pressure experiments [10-13] may consequently result from particular high strain hardening or high stresses as observed in nanoindenter experiments [9]. Such conditions remain out of the scope of the present study dedicated to the early stage of plasticity.

Not surprisingly, the pressure evolution of screw dislocation cores cannot be predicted by the classical, 1D, Peierls-Nabarro model $[28,29]$ that is intrinsically restricted to the study of planar cores [70]. Even so, our current results on edge dislocations spreading are found in good agreement with 1D PN approach [29], confirming that the PN model remains a powerful tool to evaluate core spreading of planar edge dislocations as soon as $\gamma$-surfaces are used as input $[44,72]$.

The screw dislocation core structures suggest a slip system inversion triggered by pressure. However, we need to consider the simultaneous influence of pressure and temperature on the mobility of the various dislocation characters. This can be appraised by the evolution with pressure of the critical enthalpy for kink pair nucleation $\Delta H^{*}(\tau)$. Figure 5 shows that in $\{110\}$, the motion of screw dislocations is always rate controlling. Under the influence of the core 
spreading outside $\{110\}$, both the Peierls stress and $\Delta H_{0}$ evolve strongly with pressure. In comparison, the edge dislocations also harden, but at a more moderate rate. The situation is different on $\{100\}$. For $1 / 2<110>\{100\}$ screw dislocations, the elastic hardening with increasing pressure is obviously compensated by the increasing ease of glide resulting from the core evolution. Consequently, the edge component becomes rate controlling over the whole stress range at high pressure. Comparing these two rate controlling dislocations, it is worth noticing that their $\Delta H_{0}$ converge toward a very similar value at $100 \mathrm{GPa}$, whereas the Peierls stress increases much more for the screw $1 / 2<110>\{110\}$ dislocations than for the edge $1 / 2<110>\{100\}$.

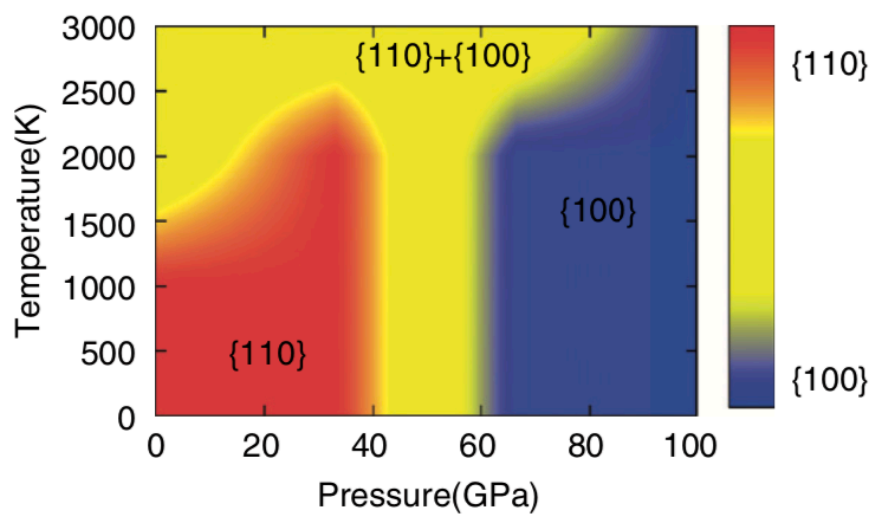

Figure 7. Predicted slip system activities in $\mathrm{MgO}$ as a function of pressure and temperature. Red and blue areas correspond to $1 / 2\langle 110\rangle\{110\}$ and $1 / 2\langle 110\rangle\{100\}$, respectively, as dominant slip systems; in between, the yellow area defines $(P, T)$ regions where CRSS for the both slip systems are of the same magnitude.

Having identified the rate controlling dislocations, it is possible to incorporate the relevant mobility into the Orowan equation. For a given strain-rate (here taken as $10^{-4} \mathrm{~s}^{-1}$ and for a typical dislocation density $\rho$ of $10^{12} \mathrm{~m}^{-2}$ ) one can calculate the CRSS as a function of $T$ (Figure 6). It is shown that pressure has several effects, the slip system inversion previously described being the first one. This phenomenon can be summarized in $P, T$ space (Figure 7). Below $2000 \mathrm{~K}$, the transition starts at ca. $30 \mathrm{GPa}$. Above ca. $60 \mathrm{GPa}$, the deformation becomes dominated by slip on $\{100\}$. In between, both slip systems exhibit comparable activity, as it is also the case at very high temperature. Slip system activities inferred from Figure 7 are consistent with the initial suggestion of Karato [16] that $1 / 2<110>\{100\}$ should be favoured at high pressure but not with texture analysis of $\mathrm{MgO}$ deformed under high-pressure (above $30 \mathrm{GPa}$ ) which still indicate a dominant activity of $1 / 2<110>\{110\}$ slip [22,23]. In the experiments by Merkel et al. [22], $\mathrm{MgO}$ powder was compressed to 35 and $47 \mathrm{GPa}$ which should be able to capture a slip 
system inversion. However, they note that in these experiments plastic deformation is not controlled independently from pressure and sharp textures appear rapidly (above ca. $2 \mathrm{GPa}$ ) as a consequence complete saturation is reached at 12 or $20 \mathrm{GPa}$. The same behaviour is exhibited in the experiments by Tommaseo et al. [23]: at room temperature, the dislocation density of the low-pressure slip system stored is probably too high to allow activation of the high-pressure slip system to be revealed. Deformation experiments performed above $60 \mathrm{GPa}$ (avoiding large strains gained at low pressure) are thus needed to verify the occurrence of a pressure-induced slip system change in $\mathrm{MgO}$.

The second effect of increasing pressure is to shift the athermal temperature (temperature corresponding to the end of the thermally activated regime) toward higher temperatures. Indeed, considering a CRSS below a few MPa (Figure 6), we found that for $1 / 2<110>\{110\}$ (respectively $1 / 2<110>\{100\}$ ), $T_{a}$ increases with increasing pressure from $2000 \mathrm{~K}$ (respectively $2800 \mathrm{~K}$ ) at 30 GPa up to $4400 \mathrm{~K}$ (respectively $4500 \mathrm{~K}$ ) at $100 \mathrm{GPa}$. The athermal temperatures of both slip systems become therefore quite comparable at high pressure. Consequently, unless extremely high temperatures are involved, the plastic behaviour of $\mathrm{MgO}$ at high pressure becomes strongly thermally activated and controlled by lattice friction (a very different behaviour compared to what is experienced usually for this material at ambient pressure).

The third effect is of course to harden the material since at a given temperature, the CRSS increase with pressure for both slip systems. It is very difficult to compare our calculations with experimental results since high pressure data are scarce. As already mentioned in [25], most high-pressure deformation experiments were performed at room temperature where $\mathrm{MgO}$ exhibit a very strong strain hardening beyond the yield point. Most of the time, experimental stress values are very far from our CRSS. However, the experiments of Mei et al. [26], performed at both high-pressure and high-temperature, might allow more relevant comparisons, in particular by comparing apparent activation volumes. Since, the pressure dependence of the flow stress (recorded by lattice strain measurement from diffraction on the (200) plane) is obtained at $310^{-5} \mathrm{~s}^{-1}$ and $1473 \mathrm{~K}$, we can use our CRSS calculated in similar conditions (i.e. $10^{-4} \mathrm{~s}^{-1}$ and $1500 \mathrm{~K}$ ) to compare our predicted pressure dependence with their observations. Our computed values, $V_{\{110\}}^{*} \approx 1.510^{-6} \mathrm{~m}^{3} / \mathrm{mol}$ and $V_{\{100\}}^{*} \approx 2.310^{-6} \mathrm{~m}^{3} / \mathrm{mol}$, appear to be consistent (given the uncertainties) with the experimental one of $2.410^{-6} \mathrm{~m}^{3} / \mathrm{mol}$ [26]. 


\section{Conclusion}

In this study, the hierarchical multi-scale numerical model developed to describe plastic deformation of $\mathrm{MgO}$ proposed in [31] has been extended to investigate the plastic behaviour of $\mathrm{MgO}$ under pressures representative of the Earth's mantle. Our study sheds some light on the influence of pressure on plastic deformation of this oxide.

Pressure affects bonding. In $\mathrm{MgO}$, this is shown by the strong increase of the elastic constants with increasing pressure. As originally suggested by Poirier [73], this elastic effect contributes to the general tendency of the Peierls stress and CRSS to increase with increasing pressure. Increasing pressure enhances lattice friction and widens the temperature range where thermally activated glide dominates. Consequently, one observes that the athermal temperature $T_{a}$ is shifted to higher values with increasing pressure.

In $\mathrm{MgO}$, pressure also has a more subtle effect through the core structure of dislocations. This effect is related to the elastic anisotropy of $\mathrm{MgO}$ (assessed by an anisotropy factor A which is the ratio of two shear moduli) which reverses at ca. 10-20 GPa (Table 1). The differential evolution with pressure of the $\gamma$-surfaces results in a strong evolution of the $3 \mathrm{D}$ core of screw dislocations which strongly affects their mobility. This results in a slip system inversion at high pressure where $1 / 2<110>\{100\}$ tends to be easier than $1 / 2<110>\{110\}$. Further experimental investigations of deformation of $\mathrm{MgO}$ at high pressure are needed to demonstrate this slip system change which has potential implications on the dynamics of the Earth's mantle.

\section{Acknowledgements}

Computational resources have been provided by IDRIS (project 101685). As this study is part of the Diup project, authors acknowledge the support of the French ANR program. The authors are grateful to B. Devincre (LEM-ONERA) for helpful discussions and to two anonymous referees who reviewed this work for useful guidance. 


\section{References}

[1] C. O. Hulse and J. A. Pask. J. Am. Ceram. Soc. 43 (1960) p.373.

[2] S. M. Copley and J. A. Pask. J. Am. Ceram. Soc. 48 (1965) p.139.

[3] C. W. Weaver and M. S. Paterson. J. Am. Ceram. Soc. 52 (1969) p.293.

[4] M. S. Paterson and C. W. Weaver. J. Am. Ceram. Soc. 53 (1970) p.463.

[5] M. Srinivasan and T. G. Stoebe. J. Mat. Sci. 9 (1974) p.121.

[6] J. L. Routbort. Acta Metallurgica 27 (1979) p.649.

[7] P. Haasen, C. Barthel, and T. Suzuki. in Dislocations in Solids, edited by H. Suzuki, T.

Ninomiya, K. Sumino, and S. Takeuchi (University of Tokyo Press, 1985), p. 455.

[8] J.P. Hirth and J. Lothe. Theory of dislocations, McGraw-Hill, New York (1968).

[9] C. Tromas, J. C. Girard, and J. Woirgard. Phil. Mag. A 80 (2000) p.2325.

[10] I. Stretton, F. Heidelbach, S. Mackwell, and F. Langenhorst. Earth and Planetary Science Letters 194 (2001) p.229.

[11] L. Li, D. J. Weidner, J. Chen, T. Vaughan, M. Davis, and W. B. Durham. J. Applied Physics 95 (2004) p.8357.

[12] D. Yamazaki and S. Karato. Phys. Earth and Planetary Int. 131 (2002) p.251.

[13] E. Mariani, J. Mecklenburgh, J. Wheeler, D. J. Prior, and F. Heidelbach. Acta Mater. 57 (2009) p.1886.

[14] G. Fontaine and P. Haasen. Physica Status Solidi b 31 (1969) K67.

[15] A. Foitzik, W. Skrotzki, and P. Haasen. Mater. Sci. Eng. A 113 (1989) p.399.

[16] S. Karato. Earth Planets Space 50 (1998) p.1019.

[17] T. A. Auten and S. V. Radcliffe. J. Am. Ceram. Soc. 59 (1976) p.249.

[18] T. A. Auten, S. V. Radcliffe, and R. B. Gordon. J. Am. Ceram. Soc. 59(1976) p.40.

[19] G. L. Kinsland and W. A. Bassett. J. Applied Physics 48 (1977) p.978.

[20] C. Meade and R. Jeanloz. J. Geophys. Res. B 93 (1988) p.3261.

[21] T. S. Duffy, R. J. Hemley, and H. Mao. Phys. Rev. Lett. 74 (1995) p.1371.

[22] S. Merkel, H. R. Wenk, J. Shu, G. Shen, P. Gillet, H. K. Mao, and R. J. Hemley. J. Geophys. Res. 107 (2002) p.2271.

[23] C. E. Tommaseo, J. Devine, S. Merkel, S. Speziale, and H. R. Wenk. Phys. Chem. Min. 33 (2006) p.84.

[24] Y. Wang, W. B. Durham, I. C. Getting, and D. J. Weidner. Review of Scientific Instruments 74 (2003) p.3002. 
[25] T. Uchida, Y. Wang, M. L. Rivers, and S. R. Sutton. Earth and Planetary Science Letters 226 (2004) p.117.

[26] S. Mei, D. L. Kohlstedt, W. B. Durham, and L. Wang. Phys. Earth and Planetary Int. 170 (2008) p.170.

[27] C. H. Woo and M. P. Puls. Phil. Mag. 35 (1977) p.1641.

[28] C. R. Miranda and S. Scandolo. Comput. Phys. Commun. 169 (2005) p.24.

[29] P. Carrez, D. Ferré, P. Cordier. Model. Simul. Mater. Sci. Engin. 17 (2009) p.035010.

[30] L. Xiong and Y. Chen. Inter. journal of Solids and Structures 46 (2009) p.1448.

[31] J. Amodeo, P. Carrez, and P. Cordier. Acta Mater. 59 (2011) p.2291.

[32] C. Denoual. Phys. Rev. B 70 (2004) p.024106.

[33] R. E. Peierls. Proc. Phys. Soc. Lond. 52 (1940) p.34.

[34] F. R. N. Nabarro. Proc. Phys. Soc. Lond. 59 (1947) p.256.

[35] C. Denoual. Comput. Methods Appl. Mech. Engrg 196 (2007) p.1915.

[36] L. Pillon, C. Denoual, and Y. P. Pellegrini. Phys. Rev. B 76 (2007) p.224105.

[37] L. Pillon and C. Denoual. Philos. Mag. 89 (2009) p.127.

[38] A. Metsue, P. Carrez, C. Denoual, D. Mainprice, and P. Cordier. Acta Mater. 58 (2010) p.1467.

[39] L. Giacomazzi, P. Carrez, S. Scandolo, and P. Cordier. Phys. Rev. B 83 (2011) p.014110. [40] V. V. Bulatov and E. Kaxiras. Phys. Rev. Lett. 78 (1997) p.4221.

[41] G. Lu. in Handook of Materials Modelling, Ed. by S. Yip, Springer Netherlands. (2005) p. 793.

[42] A. H. W. Ngan. J. Mech. Phys. Solids 45 (1997) p.903.

[43] A. H. W. Ngan and H. F. Zhang. J. Applied Physics 86 (1999) p.123.

[44] V. Vitek. Phil. Mag. 18 (1968) p.773.

[45] G. Kresse and J. Hafner. Phys. Rev. B 47 (1993) p.558.

[46] G. Kresse and D. Joubert. Phys. Rev. B 59 (1999) p.1758.

[47] J. Cai, C. Lu, P. H. Yap, and Y. Y. Wang. Applied Physics Letters 81 (2002) p.3543.

[48] J. Durinck, A. Legris, and P. Cordier. Am. Mineral. 90 (2005) p.1072.

[49] B. B. Karki, L. Stixrude, S. J. Clark, M. C. Warren, G. J. Ackland, and J. Crain. Am. Mineral. 82 (1997) p.51.

[50] B. B. Karki, R. M. Wentzcovitch, S. de Gironcoli, and S. Baroni. Phys. Rev. B 61 (2000) p.8793.

[51] S. N. Luo, D. C. Swift, R. N. Mulford, N. D. Drummond, and G. J. Ackland. J. Phys. : Condens. Matter 16 (2004) p.5435. 
[52] F. Appel and B. Wielke. Mater. Sci. Eng. 73 (1985) p.97.

[53] H. Koizumi, H. O. K. Kirchner, and T. Suzuki. Acta metall. mater. 41 (1993) p.3483.

[54] H. Koizumi, H. O. K. Kirchner, and T. Suzuki. Phil. Mag. A 69 (1994) p.805.

[55] P. Carrez and P. Cordier. in Reviews in Mineralogy \& Geochemistry, Vol. 71, edited by M. S. o. America (2010).

[56] P. Guyot and J. E. Dorn. Canadian J. of Physics 45 (1967) p.983.

[57] J. E. Dorn and S. Rajnak. Trans. Met. Soe. AIME 230 (1964) p.1052.

[58] B. Devincre, L. P. Kubin, C. Lemarchand and R. Madec. Mater. Sci. Eng. A 309-310 (2001) p.211.

[59] M. Tang, B. Devincre, and L. P. Kubin. Acta Mater. 46 (1998) p.3221.

[60] G. Monnet, B. Devincre, and L. P. Kubin. Acta Mater. 52 (2004) p.4317.

[61] S. Naamane, G. Monnet, and B. Devincre. Int. J. of Plasticity 26 (2010) p.84.

[62] D. Rodney, Phys. Rev. B 76 (2007) p.144108.

[63] B. Joos and M. S. Duesbery. Phys. Rev. Lett. 78 (1997) p.266.

[64] B. Joos and J. Zhou. Phil. Mag. A 81 (2001) p.1329.

[65] U. F. Kocks, A. S. Argon, and M. F. Ashby. Thermodynamics and kinetics of slip, Vol. 19, Pergamon Press (1975).

[66] F. Louchet. J. Mater. Sci. 41 (2006) p.2641.

[67] V. Belzner and F. Granzer. Physica Status Solidi a 39 (1977) p.183.

[68] V. V. Bulatov, O. Richmond, and V. Glazov. Acta Mater. 47 (1999) p.3507.

[69] L. H. Yang, P. Soderlind, and J. A. Moriarty. Mater. Sci. Eng. A 309-310 (2001) p102.

[70] L. Pizzagalli, J.-L. Demenet, and J. Rabier. Phys. Rev. B 79 (2009) p.045203.

[71] G. Schoeck. Mat. Sci. and Eng. A 400-401 (2005) p.7.

[72] B. Joos, Q. Ren, and M. S. Duesbery. Phys. Rev. B 5 (1994) p.5890.

[73] J. P. Poirier. Creep of crystals: High-temperature deformation processes in metals, ceramics and minerals, Cambridge University Press, Cambridge (1985). 